\title{
Production d'hydrogène au moyen d'un cycle thermo-électrochimique mettant en œuvre l'énergie solaire
}

\author{
D. Steinmetz, R. Routie et A. C. Vialaron \\ C.N.R.S.-P.I.R.D.E.S. Institut du Génie Chimique, \\ Chemin de la Loge, 31078 Toulouse Cedex, France \\ (Reçu le 14 juin 1978, révisé le 23 octobre 1978, accepté le 27 octobre 1978)
}

\begin{abstract}
Résumé. - La production d'hydrogène au moyen d'un cycle thermo-électrochimique est envisagée. L'énergie solaire est utilisée pour décomposer $\mathrm{MgSO}_{4}$ en $\mathrm{MgO}, \mathrm{SO}_{2}$ et $\mathrm{O}_{2}$. Un appoint d'électricité permet de réaliser l'oxydation électrochimique des sulfites en sulfates avec production d'hydrogène sous une différence de potentiel bien inférieure à celle nécessitée par l'électrolyse classique. Un schéma d'installation est proposé. La faisabilité des différentes étapes élémentaires du cycle est démontrée.
\end{abstract}

Abstract. - A thermo-electrochemical cycle is considered for hydrogen production. Solar energy is used for decomposition of $\mathrm{MgSO}_{4}$ to $\mathrm{MgO}, \mathrm{SO}_{2}$ and $\mathrm{O}_{2}$. Electricity is supplied to realize electrochemical oxidation of sulfites to sulfates with hydrogen production. The electrical voltage is much less than for classical electrolysis. A flow-sheet is proposed. The feasibility of each step is demonstrated.

Introduction. - La variabilité du rayonnement solaire, l'impossibilité de faire concorder l'ensoleillement aux heures et lieux de la demande énergétique obligent à résoudre le problème du stockage. Le stockage thermique à long terme d'importantes quantités d'énergie est difficile, et malgré les études en cours (batterie sodium-soufre, accumulateur zincair) le stockage électrique n'est pas encòre performant. Une perspective attrayante serait de fabriquer de l'hydrogène, ce qui résoudrait simultanément le problème du stockage de longue durée et du transport vers les zones d'utilisation peu ensoleillées.

La production d'hydrogène au moyen d'énergie solaire peut s'envisager de plusieurs manières :

a) Par voie électrochimique :

- électrolyse classique

- électrolyse à haute température $\left(900{ }^{\circ} \mathrm{C}\right)$ sur électrolyte solide

- photo-électrolyse.

L'électricité et l'apport thermique éventuel étant produits par l'énergie solaire (centrales thermodynamiques ou photovoltaïques).

b) Par voie thermochimique :

- dissociation directe de l'eau à très haute température

- cycle thermochimique mettant en œuvre une série de réactions à des niveaux thermiques modérés. c) Par voie thermo-électrochimique :

- association d'une électrolyse à faible potentiel et d'une ou plusieurs réactions thermochimiques.

Nous présentons ici un cycle de ce type adapté aux contingences de l'énergie solaire.

1. Présentation du cycle thermo-électrochimique au sulfate de magnésium. - Les cycles thermo-électrochimiques sont tous caractérisés par l'association d'une étape électrochimique où la production d'hydrogène à la cathode se fait conjointement à l'oxydation d'une espèce chimique donnée, et d'une étape thermochimique où se réalise la dissociation de cette espèce oxydée avec libération d'oxygène. La dépense d'enthalpie libre au niveau de l'électrolyse sera inférieure à celle nécessaire à l'électrolyse directe de l'eau $\left(\Delta G_{298 \mathrm{~K}, \mathrm{H}_{2} \mathrm{O} \text { liq }}^{0}=56,7 \mathrm{kcal} / \mathrm{mole}\right)$; l'appoint étant évidemment fourni par apport d'enthalpie au niveau de la réaction endothermique de décomposition.

Quelques cycles thermo-électrochimiques ont déjà été proposés :

- cycle $\mathrm{SO}_{2}, \mathrm{H}_{2} \mathrm{SO}_{4}$ étudié par l'EURATOM

à Ispra (Mark 11) et la société Westinghouse [1] ;

- cycle au cadmium [2]

- cycle $\mathrm{SO}_{2}, \mathrm{H}_{2} \mathrm{SO}_{4}, \mathrm{HBr}$ (Mark 13, Ispra).

Le cycle que nous étudions [3] se caractérise par la décomposition à haute température d'un sulfate 
métallique, en l'occurrence du sulfate de magnésium, et l'électrolyse à basse température d'une solution de sulfite acide avec production d'hydrogène à la cathode et de sulfate acide à l'anode :

$\mathrm{MgSO}_{4} \stackrel{1150^{\circ} \mathrm{C}}{\longrightarrow} \mathrm{MgO}+\mathrm{SO}_{2}+1 / 2 \mathrm{O}_{2}$

avec $\Delta H=87 \mathrm{kcal} / \mathrm{mole}[4]$ entre 900 et $1150^{\circ} \mathrm{C}$ et

$\mathrm{HSO}_{3}^{-}+\mathrm{H}_{2} \mathrm{O} \rightarrow \mathrm{HSO}_{4}^{-}+\overrightarrow{\mathrm{H}_{2}}$

avec $\Delta G_{298 \mathrm{~K}}^{0}=2,7 \mathrm{kcal} / \mathrm{mole}$ [5].

Dans l'ensemble des sulfates métalliques, le magnésium a été choisi pour un certain nombre de raisons :

- la température de décomposition compatible avec la technologie solaire actuellement développée (four à cavité et centrale à tour par exemple) ;

- la faible masse atomique du magnésium;

- la possibilité de fabriquer une solution électrolytique contenant une très forte concentration d'anhydride sulfureux sous forme de bisulfite à pression atmosphérique. De plus, sous réserve de certaines précautions, ces solutions sont stables à long terme et peuvent permettre un stockage de l'énergie solaire [6] ;

- le faible prix et l'abondance du soufre et des composés magnésiens.

2. Mise en auvre et études préliminaires. - Le cycle au sulfate de magnésium sera mis en œuvre selon le schéma figure 1. La faisabilité des différentes étapes élémentaires associées dans le cycle a été démontrée par les données tirées de la littérature ou les expériences réalisées au laboratoire.

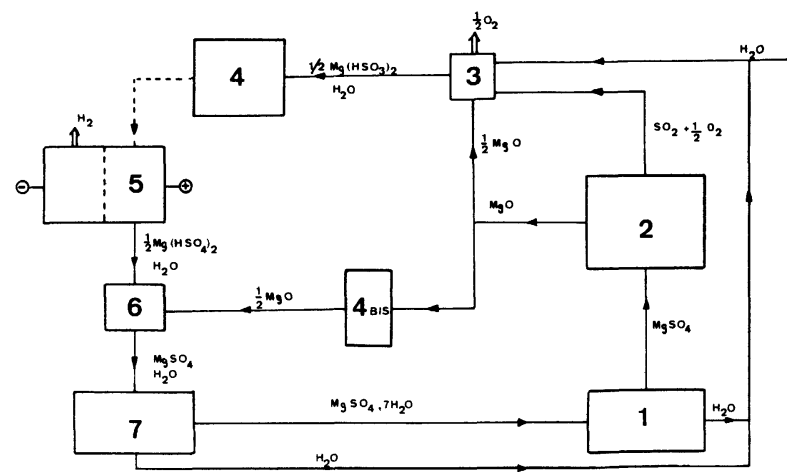

Fig. 1. - Schéma de l'installation : 1 : séchoir; 2 : four solaire 3 : séparateur ; 4 : réservoirs de stockage ; 5 : électrolyseur ; $6:$ neutraliseur ; 7 : cristalliseur.

[Flow-sheet : $1:$ dryer ; 2 : solar furnace ; 3 : separator $; 4:$ storagetanks ; 5 : electrolyser ; 6 : neutralization reactor ; 7 : crystallization reactor.]

2.1 Partie thermochimiQue. - Réacteur 1 : déshydratation de $\mathrm{MgSO}_{4}, 7 \mathrm{H}_{2} \mathrm{O}$.

Le sulfate de magnésium cristallise sous forme heptahydratée à la sortie de l'étape électrochimique.
Ce produit peut être déshydraté à des températures inférieures à $400{ }^{\circ} \mathrm{C}$, sous pression atmosphérique, comme le montrent les courbes d'analyse thermique pondérale et différentielle réalisées à la thermobalance (Fig. 2).

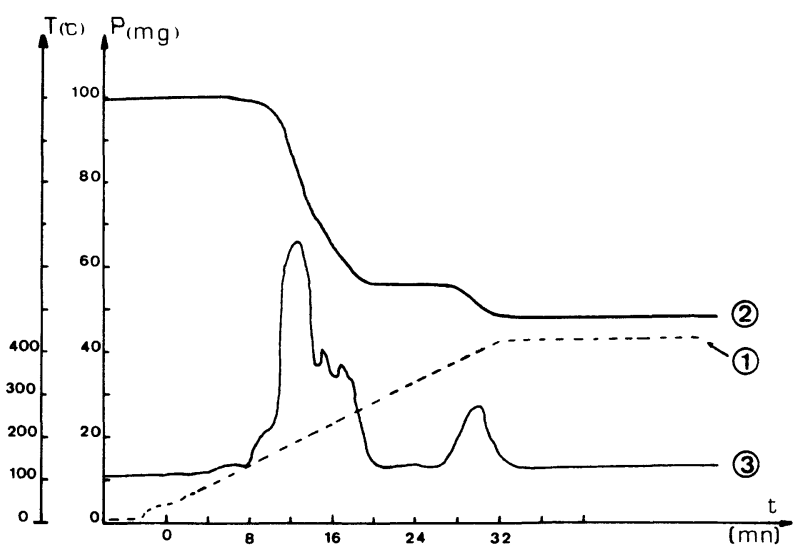

Fig. 2. - Déshydratation de $\mathrm{MgSO}_{4}, 7 \mathrm{H}_{2} \mathrm{O}$ : Courbe 1 : température ; Courbe 2 : analyse thermique pondérale; Courbe 3 : analyse thermique différentielle.

$\left[\mathrm{MgSO}_{4}, 7 \mathrm{H}_{2} \mathrm{O}\right.$ deshydratation : 1 : temperature curve ; 2 : gravimetric thermal analysis curve; 3 : differential thermal analysis curve.]

On peut estimer la chaleur nécessaire pour effectuer la déshydratation et porter les produits de réaction de 25 à $400{ }^{\circ} \mathrm{C}$ à environ $130 \mathrm{kcal} / \mathrm{mole}$, connaissant les enthalpies standard de formation ainsi que les capacités calorifiques des différentes espèces :

$$
\Delta H_{298 \mathrm{~K}}^{0} \quad-\quad C_{\mathrm{p}}
$$

$\mathrm{MgSO}_{4}, 7 \mathrm{H}_{2} \mathrm{O}-808 \mathrm{kcal} / \mathrm{mole}$

$$
\mathrm{MgSO}_{4} \quad-306 \mathrm{kcal} / \mathrm{mole} \quad 26,7 \mathrm{cal} /{ }^{\circ} \mathrm{C} \text { mole }
$$$$
\mathrm{H}_{2} \mathrm{O} \text { vapeur } \quad-57,8 \mathrm{kcal} / \mathrm{mole} \quad 0,47 \mathrm{cal} /{ }^{\circ} \mathrm{C} \mathrm{g}
$$

- Réacteur 2 : décomposition de $\mathrm{MgSO}_{4}$.

Le sulfate de magnésium anhydre est porté à haute température dans un four solaire à cavité. Dans le domaine où la décomposition a lieu, la variation d'enthalpie libre de la réaction a été calculée par Dewing et Richardson [4] :

$$
\begin{aligned}
& \mathrm{MgSO}_{4} \rightarrow \mathrm{MgO}+\mathrm{SO}_{2}+1 / 2 \mathrm{O}_{2} \\
& \Delta G_{900-1150^{\circ} \mathrm{C}}^{0}=87180-60,2 \mathrm{~T}\left(\mathrm{cal} /{ }^{\circ} \mathrm{C} . \mathrm{mole}\right) .
\end{aligned}
$$

A ces températures la dissociation de $\mathrm{SO}_{3}$ est totale. Une trempe des gaz sera nécessaire pour éviter toute recombinaison.

$\mathrm{Au}$-dessus de $1150^{\circ} \mathrm{C}, \mathrm{MgSO}_{4}$ fond en se décomposant. La cinétique de décomposition a été étudiée à la thermobalance (Fig. 3). Cependant, ces expériences, effectuées dans des conditions rigoureusement isothermes pour de faibles quantités de matière $\left(100 \mathrm{mg}\right.$ de $\left.\mathrm{MgSO}_{4}\right)$ et en présence d'un balayage 
d'argon, ne préjugent pas du fonctionnement réel au four solaire. Des essais dans des conditions proches de la réalité (four $1000 \mathrm{~kW}$ d'Odeillo) sont prévus. Ils permettront en particulier de déterminer le taux de conversion en fonction des conditions opératoires et notamment du temps de séjour.

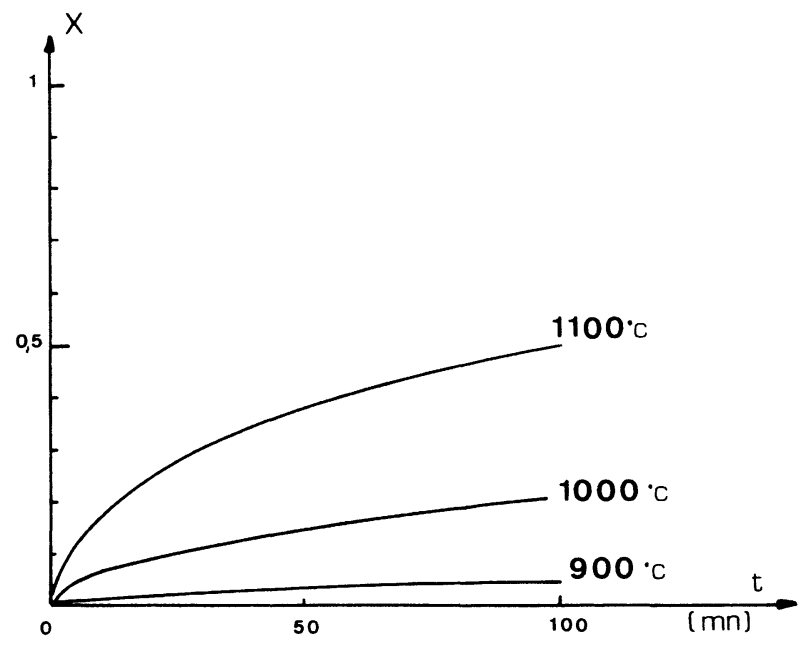

Fig. 3. - Décomposition isotherme de $\mathrm{MgSO}_{4}$.

[ $\mathrm{MgSO}_{4}$ isothermal decomposition.]

L'importance relative des différentes étapes de la partie thermochimique est mise en évidence sur le diagramme $\Delta H, T$ de la figure 4 . La déshydratation représente près de la moitié des besoins thermiques du cycle, et la pénalise fortement tant que l'on ne récupère pas l'énergie contenue dans la vapeur d'eau produite à $400{ }^{\circ} \mathrm{C}$.

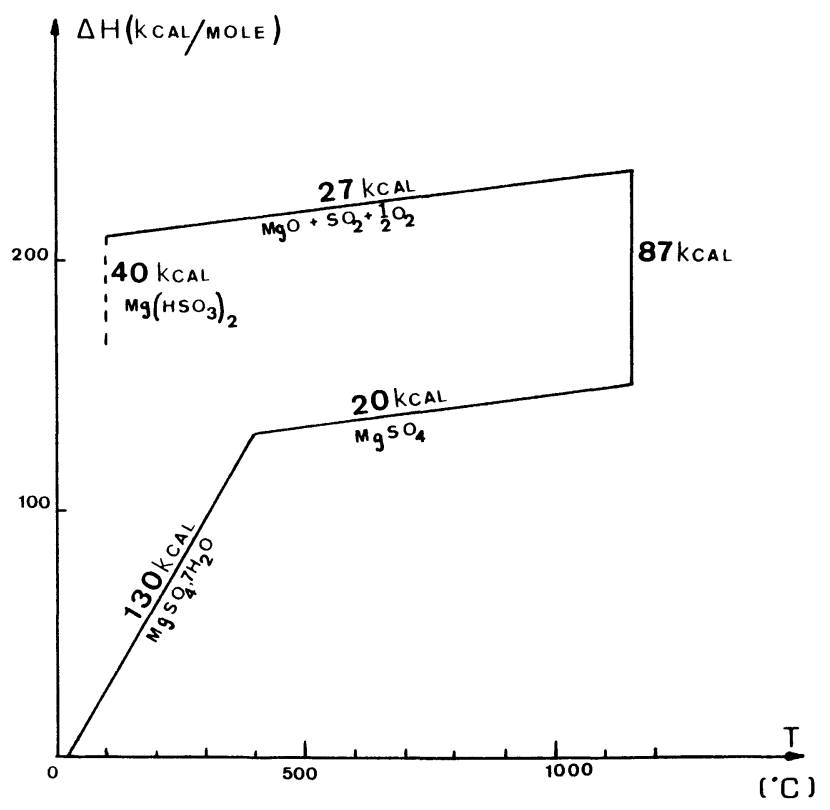

Fig. 4. - Diagramme $\Delta H, T$ de la partie thermochimique du cycle. [ $\Delta H, T$ diagram for thermochemical steps of the cycle.]
2. 2 SÉparation $\mathrm{SO}_{2} / \mathrm{O}_{2}$. Stockage. - Réacteur 3 : séparation $\mathrm{SO}_{2} / \mathrm{O}_{2}$.

Après récupération des chaleurs sensibles des produits de décomposition, la moitié de la magnésie est envoyée au stockage 4 bis, l'autre moitié sera mise en solution dans le réacteur 3 où s'effectuent conjointement la séparation $\mathrm{SO}_{2}-\mathrm{O}_{2}$ et la préparation de la solution de bisulfite de magnésium qui sert d'électrolyte :

$\mathrm{MgO}+\mathrm{H}_{2} \mathrm{O}+2 \mathrm{SO}_{2} \rightarrow \mathrm{Mg}\left(\mathrm{HSO}_{3}\right)_{2}$.

La récupération de l'anhydride sulfureux par la magnésie en solution aqueuse est un procédé couramment utilisé [7], tant par l'industrie papetière que pour des traitements de dépollution. Une grande quantité d'anhydride sulfureux peut passer en solution aqueuse sous forme $\mathrm{Mg}\left(\mathrm{HSO}_{3}\right)_{2}$ jusqu'à $500 \mathrm{~g}$ de $\mathrm{SO}_{2} /$ litre d'eau à $25^{\circ} \mathrm{C}$ [8]. L'oxygène étant peu réactif vis-à-vis de ces solutions si la température est inférieure à $30^{\circ} \mathrm{C}$, on peut espérer que la séparation par absorption sélective sera un procédé efficace et énergétiquement économique, par rapport à la séparation $\mathrm{SO}_{2} / \mathrm{O}_{2}$ par compression et liquéfaction préconisée par Westinghouse.

- Bac de stockage 4

Sous réserve de certaines précautions la solution de bisulfite obtenue est stable chimiquement. La capacité volumique de ce stockage est particulièrement intéressante : $1 \mathrm{~m}^{3}$ de solution permet de stocker 8000 moles de $\mathrm{SO}_{2}\left(\simeq 179 \mathrm{~m}^{3} \mathrm{TPN}\right)$, c'est-à-dire l'équivalent en hydrogène après une électrolyse à potentiel modéré.

2.3 Partie ÉlectrochimiQue. - Réacteur 5 : l'électrolyseur.

L'électrolyte sortant du stockage 4 est envoyé dans le compartiment anodique de l'électrolyseur 5 où s'effectue l'oxydation du sulfite acide en sulfate acide :

$\mathrm{HSO}_{3}^{-}+\mathrm{H}_{2} \mathrm{O} \rightarrow \mathrm{HSO}_{4}^{-}+2 \mathrm{H}^{+}+2 \mathrm{e}$

$\operatorname{avec} E=0,059-0,059 \mathrm{pH}+0,0295 \log \frac{\left(\mathrm{HSO}_{4}^{-}\right)}{\left(\mathrm{HSO}_{3}^{-}\right)} V$

les $\mathrm{H}^{+}$formés migrent vers la cathode où ils sont réduits :

$2 \mathrm{H}^{+}+2 \mathrm{e} \rightarrow \mathrm{H}_{2}^{\top}$

avec $E=-0,059 \mathrm{pH} V$.

L'électrolyseur 5 comportera deux compartiments séparés par un diaphragme ou une membrane semiperméable de façon à empêcher toute diffusion des espèces anioniques $\mathrm{HSO}_{3}^{-}$vers la cathode et à éviter ainsi de réduire les sulfites en soufre, réaction qui viendrait concurrencer la production d'hydrogène et polluer la cathode. L'oxydation électrochimique des sulfites est une réaction fortement irréversible 
comme le montre la figure 5. Afin de diminuer les surtensions de réaction on pourra augmenter la température et utiliser comme électrode des matériaux activés à grande surface spécifique (grains de carbone platiné par exemple). On peut espérer dans ces conditions, en tenant compte des surtensions anodique et cathodique, et de la chute ohmique (principalement dans la membrane), effectuer l'électrolyse avec des différences de potentiel pratiques de l'ordre de 0,7 Volt pour une intensité de $1000 \mathrm{~A} / \mathrm{m}^{2}$ de membrane.

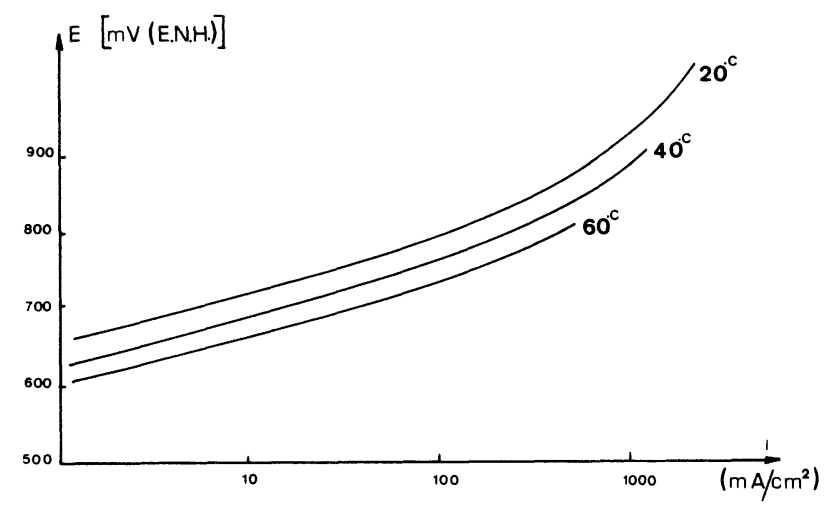

Fig. 5. - Courbe de polarisation anodique potentiostatique, relative à l'oxydation sur platine de $\mathrm{Mg}\left(\mathrm{HSO}_{3}\right)_{2} 1 \mathrm{~mol} / 1$ (électrode à disque, vitesse de rotation $1000 \mathrm{~T} / \mathrm{min}$ ).

platinum disc electrode (rotation speed 1000 r.p.m.).]

- Réacteur 6 : neutralisation de $\mathrm{Mg}\left(\mathrm{HSO}_{4}\right)_{2}$.

A la sortie de l'électrolyseur, la solution contiendra donc du sulfate acide de magnésium qui sera neutralisé par la magnésie du stockage 4 bis :

$$
\mathrm{Mg}\left(\mathrm{HSO}_{4}\right)_{2}+\mathrm{MgO} \rightarrow 2 \mathrm{MgSO}_{4}+\mathrm{H}_{2} \mathrm{O}
$$

- Réacteur 7 : cristallisation de $\mathrm{MgSO}_{4}$.

A la sortie du réacteur 6 l'électrolyte contenant des cations $\mathrm{Mg}^{++}$, des anions $\mathrm{HSO}_{3}^{-}$provenant du bisulfite non oxydé et des anions $\mathrm{SO}_{4}^{-}$, est refroidi jusqu'à une température de l'ordre de $20^{\circ} \mathrm{C}$ pour séparer le sulfate du sulfite acide de magnésium. En effet, lorsque la température diminue, la solubilité du sulfite augmente et celle du sulfate diminue, de sorte qu'à $20^{\circ} \mathrm{C}$ le sulfate de magnésium cristallise sous forme $\mathrm{MgSO}_{4}, 7 \mathrm{H}_{2} \mathrm{O}$. La solution aqueuse restante peut être recyclée à l'entrée de l'électrolyseur 5 . ou au niveau du réacteur 3 (séparation $\mathrm{SO}_{2} / \mathrm{O}_{2}$ ).

3. Rendement et intérêt du cycle. - On peut estimer le rendement thermique de ce cycle en adoptant la définition du rendement proposée par Funk [9] :

$\eta=\frac{\Delta H_{298 \mathrm{~K}, \mathrm{H}_{2} \mathrm{O} \mathrm{liq}}^{0}}{Q_{1}+\frac{W}{\varepsilon}-Q_{2}}$

avec $\Delta H_{298 \mathrm{~K}, \mathrm{H}_{2} \mathrm{O} \text { liq }}^{0}=68,3 \mathrm{kcal} / \mathrm{mole}$
$Q_{1}=$ chaleur totale à fournir

$Q_{2}=$ chaleur récupérable (affectée d'un coefficient de récupération)

$W=$ travail à fournir

$\varepsilon=$ coefficient de conversion de la chaleur en travail, on choisit $\varepsilon=0,22$ rendement du cycle thermodynamique de la centrale THEMIS (dans le cas d'un système tout solaire). On ne tient pas compte à ce niveau des rendements du réacteur solaire et des pertes par réflexions sur les miroirs.

Dans le cas du cycle au sulfate de magnésium étudié, le rendement s'exprime en fonction du taux de conversion du four solaire $X$ (la déshydratation est totale), de la tension d'électrolyse $V$ et du pourcentage de chaleur récupérée $Y$. Par mole de $\mathrm{MgSO}_{4}$ effectivement décomposée, les besoins en chaleur du cycle seront :

$Q_{1}=\frac{150}{X}+87 \mathrm{kcal}$ (cf. Fig. 4) .

On suppose pouvoir récupérer les calories jusqu'à un niveau thermique de $100^{\circ} \mathrm{C}$. $Q_{2}$ est la somme de :

- l'énergie de la vapeur d'eau récupérée à la déshydratation, entre 400 et $100^{\circ} \mathrm{C}$, y compris la chaleur de condensation : soit $86 \mathrm{kcal}$ par mole de $\mathrm{MgSO}_{4}$, $7 \mathrm{H}_{2} \mathrm{O}$ traité ;

- la chaleur sensible des produits de décomposition de $\mathrm{MgSO}_{4}\left(\right.$ de 1150 à $100{ }^{\circ} \mathrm{C}$ ) : soit $27 \mathrm{kcal}$ par mole de $\mathrm{MgSO}_{4}$ décomposé ;

- la chaleur sensible de $\mathrm{MgSO}_{4}$ non décomposé (de 1150 à $100^{\circ} \mathrm{C}$ ) : soit $28 \mathrm{kcal}$ par mole de $\mathrm{MgSO}_{4}$.

On considère d'autre part que la chaleur de formation du bisulfite en solution aqueuse n'est pas récupérable :

$Q_{2}=\frac{86}{X}+27+\left(\frac{1}{X}-1\right) 28 \mathrm{kcal}$.

Le travail $W$ est égal à l'énergie dépensée par l'électrolyse, les autres travaux étant supposés négligeables.

$W=2 \frac{96,5}{4,18} V \mathrm{kcal}$

d'où en pratique

$\eta=\frac{68,3}{\frac{150}{X}+87+209 V-Y\left(\frac{114}{X}-1\right)}$

On peut comparer ce rendement à celui de l'électrolyse directe de l'eau (centrale THEMIS et électrolyse sous $1,8 \mathrm{~V}$ )

$\eta^{*}=\frac{68,3}{209 \times 1,8}=0,18$.

La figure 6 représente pour un rendement des échangeurs donné $(Y=0,7)$ la variation de $\eta$ en fonction de $X$ et de $V$. 


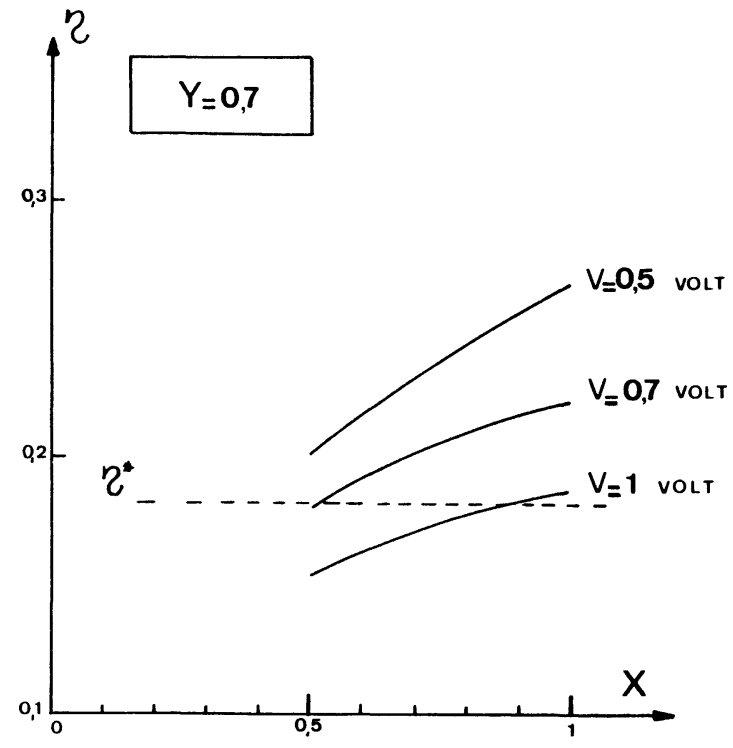

Fig. 6. - Rendement du cycle thermo-électrochimique.

[Efficiency of the thermo-electrochemical cycle.]

Le cycle hybride au sulfate de magnésium n'a un rendement supérieur à l'électrolyse classique que pour un taux de conversion du four solaire satisfaisant et une tension d'électrolyse inférieure à $0,7 \mathrm{~V}$.

On peut remarquer que l'emploi d'autres sulfates métalliques tels que calcium, baryum, strontium, au prix d'autres difficultés, aurait permis de meilleurs rendements en raison du fait qu'ils cristallisent respectivement sous forme bihydratée $\left(\mathrm{CaSO}_{4}, 2 \mathrm{H}_{2} \mathrm{O}\right)$ et anhydre $\left(\mathrm{BaSO}_{4}, \mathrm{SrSO}_{4}\right)$.

4. Comparaison aux cycles à l'acide sulfurique. On peut comparer le cycle au sulfate avec les cycles à l'acide sulfurique (Mark 11 et Westinghouse). Parmi toutes les variantes possibles nous avons choisi les conditions expérimentales suivantes :

$-\mathrm{H}_{2} \mathrm{SO}_{4}$ à $50 \%$ à l'électrolyseur. - C'est dans ce domaine de concentration que l'électrolyse s'effectuera de la manière la plus satisfaisante, avec toutefois des tensions d'électrolyse de l'ordre de 0,6 à $0,8 \mathrm{~V}[10]$.

- Une température haute de $1000^{\circ} \mathrm{C}$. - Cette température est envisageable au four solaire et permet une décomposition totale de l'anhydride sulfurique.

- Une distillation de $\mathrm{H}_{2} \mathrm{SO}_{4}$ à $50 \%$ à $123{ }^{\circ} \mathrm{C}$ avec élimination de la vapeur d'eau à cette température. A cause des problèmes de corrosion, la mise en œuvre de cette étape sera difficile, et le faible niveau thermique de la vapeur empêchera sa valorisation énergétique.

Le diagramme $\Delta H, T$ de ce cycle (Fig. 7) montre qu'au niveau des besoins thermiques le cycle à l'acide sulfurique est un peu plus intéressant. On peut dans ce cas calculer le rendement $\eta^{\prime}$. Le travail $W$ à fournir comprendra ici, en plus de l'électrolyse le travail de séparation $\mathrm{SO}_{2}-\mathrm{O}_{2}$ que l'on peut estimer dans un premier temps comme le double du travail théorique $W_{\mathrm{s}}$.

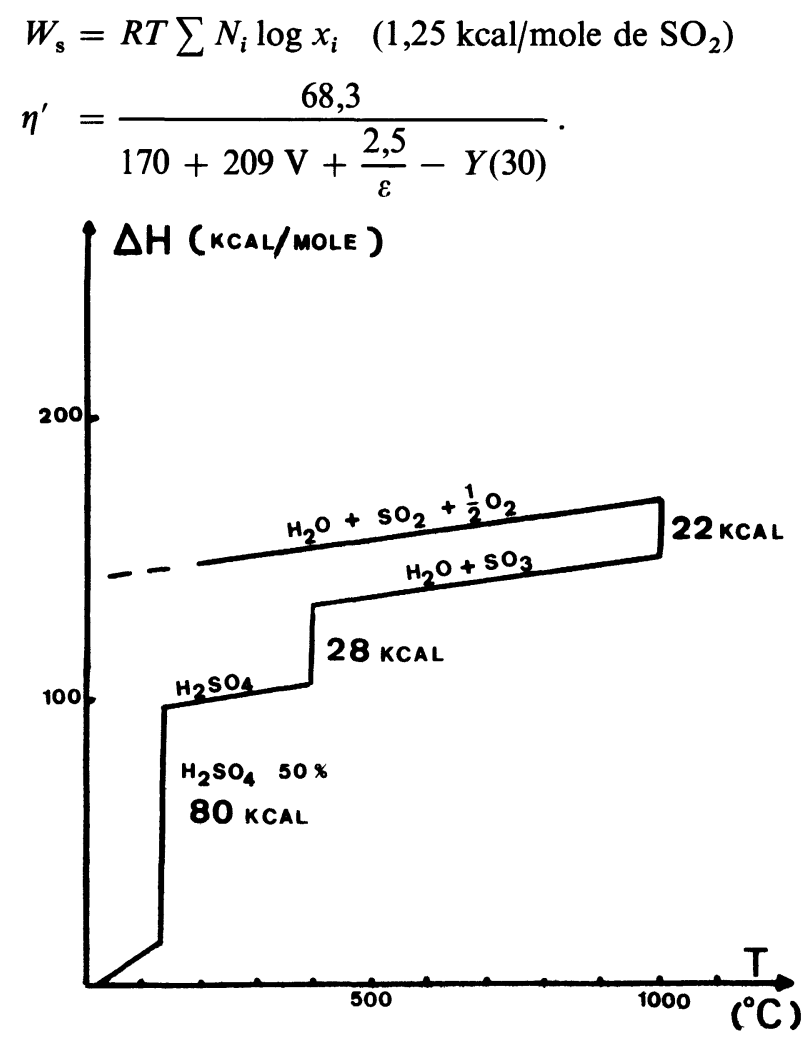

Fig. 7. - Diagramme $\Delta H, T$ du cycle hybride à $\mathrm{H}_{2} \mathrm{SO}_{4}$.

$\left[\Delta H, T\right.$ diagram for $\mathrm{H}_{2} \mathrm{SO}_{4}$ hybrid cycle.]

A même rendement des échanges de chaleurs $(Y=0,7)$ et même tension d'électrolyse $(0,7 \mathrm{~V})$ on obtient :

$\eta^{\prime}=0,225$.

Les rendements $\eta$ et $\eta^{\prime}$ ainsi calculés ne sont pas les rendements théoriques car ils prennent en compte au moins partiellement les contraintes de la mise en œuvre des procédés. On peut déjà remarquer que les deux cycles hybrides sont voisins par leur rendement mais très différents dans leur réalisation. Le cycle à l'acide sulfurique nécessite en effet la mise au point de matériaux résistants à la corrosion et capables de tenir aux aléas solaires : chocs thermiques pour le réacteur de décomposition, arrêt et mises en route répétées pour le circuit gaz. Ce cycle implique en outre un système de chauffage annexe pour la vaporisation de l'acide, préalable à la décomposition. Ce type de cycle s'adapte bien à de grosses installations fonctionnant en continu comme les centrales nucléaires, mais l'utilisation de l'énergie solaire impose une technologie simple et peu coûteuse. Cela semble le cas pour le cycle au sulfate de magnésium qui permet en plus un stockage facile des réactants à trois niveaux énergétiques : solution de bisulfite, sulfate hydraté et déshydraté et qui pourra donc supporter les problèmes majeurs du fonctionnement discontinu. 
On peut donc affirmer que le choix de l'option hydrogène solaire se fera en fonction de critères liés à l'investissement et à l'exploitation plutôt qu'en fonction des rendements thermiques.

5. Conclusion. - Comme la plupart des cycles thermochimiques, le cycle thermo-électrochimique au sulfate de magnésium entre en concurrence avec l'électrolyse classique. Il présente cependant l'intérêt de permettre un stockage " hybride " facile à mettre en cuvre sous la forme de solution concentrée de bisulfite. On peut proposer deux scénarios d'utilisation de ce cycle qui ont peut-être un certain intérêt :

- premier scénario, entièrement solaire, avec un champ de photopiles et une centrale à tour pour la thermochimie (de la taille du projet français THEMIS) : dans cette proposition le stockage hybride fournirait une adaptation entre l'énergie solaire globale reçue par les photopiles (radiation directe et diffuse) et l'énergie solaire directe reçue par les héliostats et la tour. Le cycle thermo-électrochimique permettrait donc une importante économie sur l'investissement en photopiles (par rapport à un système photopile et électrolyse classique) ;

- le deuxième scénario est proposé comme un complément solaire à la conversion d'énergie élec- trique nucléaire en hydrogène. Un grand stockage permettrait d'emmagasiner l'énergie solaire reçue par une centrale THEMIS pour la restituer vers la production d'hydrogène à un niveau soit constant, soit ajusté aux besoins.

Les différentes étapes du cycle étudié présentent de plus, par elles-mêmes, un intérêt propre. Citons par exemple :

- la décomposition des sulfates au four solaire intéresse l'industrie papetière : elle permettrait de fabriquer au moindre coût les solutions de bisulfites utilisées dans la cuisson de la cellulose. On peut, d'autre part, à l'aide de l'énergie solaire, concevoir d'autres cycles thermochimiques pour la production d'hydrogène, la température maximale n'étant plus alors limitée par le réacteur nucléaire ;

- l'oxydation électrochimique des sulfites peut permettre de valoriser les rejets industriels d'anhydride sulfureux par une production conjointe d'acide sulfurique et d'hydrogène.

Remerciements. - Les auteurs remercient Monsieur Grezes-Besset (Toulouse) et Monsieur Nada (Odeillo) pour leur contribution aux mesures d'analyse thermique pondérale.

\section{Bibliographie}

[1] BReCHER, L. E., WESTINGHOUSE ELECTRIC ' CORPORATION, U.S. Patent 3.888.750.

[2] Pangborn, J., Ist World Hydrogen Energy Conference. 1-3 mars 1976, Miami Beach. Floride U.S.A., Vol. 1, 7 A, 59.

[3] Vialaron, A. C., Steinmetz, D., Bugarel, R., Cycle thermoélectrochimique de dissociation de l'eau, Brevet ANVAR.

[4] Dewing, E. W., Richardson, F. D., Trans. Faraday Soc. 55 (1959) 611.

[5] Pourbaix, M., Atlas d'équilibres électrochimiques à $25^{\circ} \mathrm{C}$ (Gauthier-Villars Cie Ed., Paris) 1963.
[6] Vialaron, A. C., International Symposium Workshop on Solar Energy, 16-22 juin 1978, Le Caire, Egypte.

[7] Koemler, G. R., Chem. Eng. Progr. 70 (1974) 63.

[8] Conrad, F. H., Brice, D. B., Tappi 32 (1949) 222.

[9] Funk, J. E., Conger, W. L., Carty, R. H., Hydrogen Energ! Ed. by Veziroglu, T. N. (Plenum Press, New-York) 197 . part A, p. 457.

[10] Appleby, A. J., Pichon, B., 2nd World Hydrogen Energ) Conference Zurich, 1978. 